\title{
LE RELIGIEUX ET LE POLITIQUE DANS LA REVOLUTION FRANÇAISE
}

\section{RELIGION AND POLITICS IN THE FRENCH REVOLUTION}

\author{
Olivia Leboyer \\ Institut d'Etudes Politiques
}

\begin{abstract}
Recensión de / Review of : Le religieux et le politique dans la Révolution française. L'idée de régénération, Lucien Jaume, 2015, P.U.F., collection Léviathan, $163 \mathrm{p}$.
\end{abstract}

Mots clés: Révolution française, Religion, Régénération, Droits de l'homme Key words: French Revolution, Religion, Regeneration, Human Rights

Après une fine biographie intellectuelle de Tocqueville (Fayard, 2008) et un essai percutant sur la construction européenne (Qu'est-ce que l'esprit européen, Flammarion, 2010), le philosophe Lucien Jaume s'interroge sur l'énigme de la Révolution française. Sur cette période, les ouvrages abondent, mais celui-ci se distingue tout particulièrement. La problématique est en effet centrée sur la place du religieux dans la dynamique révolutionnaire. On sait, et l'on répète à l'envi, que la Révolution française et les promesses d'égalité qu'elle porte, n'a toujours pas trouvé son terme aujourd'hui. Il en est de même pour la question de la religion, hautement délicate, et loin d'être réglée. Comment saisir la question théologicopolitique ? Pour Lucien Jaume, le moment révolutionnaire est une véritable plaque révélatrice, comme en photographie : à travers les débats qui agitent les clubs et autres cercles, quelque chose se dit, d'essentiel, sur les liens entre le politique et le religieux. Dans le politique se mêle toujours de la croyance, du sacré. Mais, dans quelle mesure exactement et comment le comprendre?

Lucien Jaume part d'une interrogation : la Révolution affirme inaugurer une ère nouvelle mais, dans les discours des acteurs, comme une ombre portée, l'ancien monde se profile toujours. Le sous-titre de l'ouvrage "l'idée de régénération " éclaire bien ce paradoxe. Peut-on refonder un ordre social à partir d'une tabula rasa? Peut-on créer cet homme nouveau évoqué par Saint-Paul ? Non, ce désir tient du mythe, et le concept de régénération exprime cette ambiguïté : dans la Révolution, il y a rupture décisive, mais aussi refonte. Rajeunir, tout en conservant, tel serait le projet. Mais dans l'idée de "faire retour à ", la question de l'origine fait évidemment problème. Quel mythe mobilise-t-on? Par quelle opération de transsubstantiation retouverait-on, magiquement, un Paradis perdu ?

Précisément, Lucien Jaume s'attache à décrypter les discours des orateurs de l'époque, pour identifier la place qu'y tient le religieux. Il s'agit de mettre au jour l'effet de sens d'un discours, soit sa rhétorique et son rapport à ses destinataires. Dans le livre, un texte en particulier retient l'attention : 
le discours de Le Chapelier, du 29 septembre 1791, sur les clubs et sociétés populaires.Trop peu connu, ce discours est ici reproduit en annexe, ce qui nous permet de suivre l'analyse dans son déroulement complet. Le Chapelier exprime un conflit politique mais, ce conflit, il tente précisément de le déplacer, de le transcender vers un domaine plus noble: la Loi, la Constitution, les grands principes de 1789. Avec une habileté rhétorique consommée, il s'efforce d'opposer la symbolique du nouvel ordre constitutionnel à "quelques individus", quelques agitateurs. La stratégie politique consiste bien à opposer le registre noble à un groupuscule, à une faction. Et le discours s'adresse d'abord aux députés mais, plus largement, à la Nation. Le procédé se retrouvera, très similaire, jusqu'au Général de Gaulle. En parlant ainsi au nom de la Nation et des grands principes, Le Chapelier entend résoudre la question de la représentation. Et pour mener une telle stratégie rhétorico-politique, il faut que l'orateur passe par un code : la culture politique révolutionnaire, qui comprend l'idée d'égalité entre les individus, la vision atomistique et cette fameuse idée de régénération, à laquelle on recourt comme à un sésame. L'enjeu est bien alors de terminer la Révolution par l'ordre constitutionnel. Et cette vision de la Constitution impliquait-elle nécessairement le rejet de la liberté d'association ? On peut estimer que non et que la rigidité des modérés provient surtout de la lutte contre l'Ancien Régime. Contre ce modèle proposé par Le Chapelier, Lucien Jaume expose un modèle concurrent, celui de Billaud-Varenne, d'esprit organique et communautaire. Billaud-Varenne entend "recouvrer " un bonheur perdu et, dans le même temps, façonner un peuple neuf. Il ne faut pas, selon ce modèle, faire des individus, mais des citoyens.

On le voit, une lecture minutieuse et affûtée des discours éclaire bien des enjeux. Ainsi, ce postulat atomistique, ferment de la culture révolutionnaire, explique sans doute pourquoi, en France, les partis politiques n'ont jamais eu de légitimité très solide. Et pour susciter de la croyance, de la légitimité, quel plus sûr appui que le religieux ? Aussi le politique va-t-il, bien souvent, et même parfois sans le savoir, parler le langage du religieux. C'est particulièrement perceptible dans la Terreur, avec le culte de l'Etre suprême proclamé par Robespierre. La confusion joue alors à plein. Pour débusquer les traces du religieux, transcrit dans le langage politique, Lucien Jaume s'intéresse notamment, dans le débat sur la Constutution civile du clergé, à un discours de l'avocat Martineau, qui souligne que l'on a beau faire la politique de la table rase, il serait bon se trouver une religion inscrite dans les mœurs, au sens romain. De la religion, Martineau retient l'idée d'un Dieu qui observe l'homme. Et le but serait de créer une constitution civile du clergé. Il s'agirait alors de transformer le corps ancien du clergé en citoyens. L'Etat devrait ainsu contrôler l'Eglise qu'il a placée en son sein.

La rhétorique des droits de l'homme est au centre de l'analyse menée par Lucien Jaume. Particulièrement novateur et éclairant, le chapitre V, intitulé "Marx, une pensée de la régénération, voulant régénérer la Révolution ", étudie avec rigueur la rhétorique de Marx, qui aurait également eu recours à cette vision régénératrice, mais sans l'avouer explicitement. De fait, Marx pense la libération à la fois comme une nouveauté et comme une 
récupération, par l'homme, de sa nature humaine. Certes, Marx refuse de poser en héritier de la Révolution. Mais là où il est d'accord, en profondeur, avec les révolutionnaires, c'est sur cette intuition forte d'une nature humaine. Complexe, sa pensée tient que l'on doit rechercher un humanisme réel, mais que les droits de l'homme, en tant que tels, doivent être critiqués. Pour Marx, les Droits de l'homme et du citoyen ne sont jamais qu'une fiction, une illusion d'égalité qui sert à masquer une forme de domination, bien réelle. Dès lors, on retrouve la question de la communauté et du lien social à recréer. Marx s'efforce ainsi de dépasser le citoyen pour retrouver l'individu comme "humanisme réel ". C'est tout l'enjeu de A propos de la Question Juive (1843), notamment. Marx y distingue clairement l'émancipation en général et l'émancipation politique des Déclarations des droits. Par endroits, chez Marx, on entend comme des échos de Tocqueville sur la démocratie américaine. Lorsqu'il écrit, par exemple, que dans la démocratie, la religion est l'esprit de la société civile.

Religion comme esprit de la société civile? Apparent refus de la spiritualité ? Comment saisir ces intuitions, désirs contradictoires ou refoulés, dans le cadre de la démocratie actuelle ? Lucien Jaume revient, par exemple, sur l'idée de foi laïque défendue récemment encore par l'ancien Ministre Vincent Peillon, dans la lignée de Ferdinand Buisson. En effet, pour Lucien Jaume, dans cette religion laïque et diffuse, sans dogme, sans institutions et sans socle, la transcendance semble bien escamotée. Au fil de l'ouvrage, l'idée de régénération nous livre ses paradoxes et zones troubles. La vertu heuristique de ce concept est très stimulante, fonctionnant aussi bien ici que l'idée de désenchantement du monde chez Marcel Gauchet. Dans la conclusion, Lucien Jaume nous invite à transposer cette matrice de la régénération à d'autres sphères que le politique, remarquant d'ailleurs que les révolutionnaires entendaient bien souvent énoncer les axiomes des droits de l'homme et des principes fondateurs avec la limpidité d'une figure de géométrie. Les révolutionnaires s'appuient, semble-t-il, à la fois sur l'idée du droit naturel et sur une inspiration religieuse. Sous des formes diverses, le politique se met à parler le langage du religieux. Il y a bien eu, selon Lucien Jaume, une "religion de la religion de la Révolution ", la formule soulignant la mise en abîme, assez vertigineuse.

Dès lors, la question du religieux en France, aujourd'hui, se pose nécessairement. Le retour historique doit en effet nous éclairer sur les enjeux actuels de la laïcité. D'un trait pertinent, Lucien Jaume clôt l'ouvrage sur l'urgence qu'il y a à penser les rapports à instaurer entre la pensée républicaine et les visions religieuses de la société, dans leur pluralité.

Enviado el (Submission date): 6/05/2015

Aceptado el (Acceptance date): 21/05/2015 